\title{
A PRISMA-driven systematic review of data mining methods used for defects detection and classification in the manufacturing industry
}

\author{
Blanka Bártováa* (D), Vladislav Bína ${ }^{a{ }^{a *}}$ (D), Lucie Váchováa ${ }^{a * * *}$ \\ `Vysoká škola ekonomická v Praze, Fakulta managementu, Jindřichův Hradec, Czech Republic

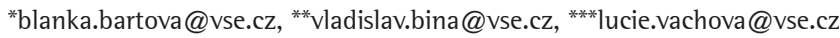

\begin{abstract}
Paper aims: This research aims to analyze the primary studies published in recent years focusing on defect detection or classification in manufacturing and extract information about frequently used data mining (DM) methods, their accuracy, strengths, and limitations.
\end{abstract}

Originality: Industrial production is now undergoing a dynamic transformation in the context of Industry 4.0, where implementation of data mining is a frequently discussed topic, and such an overall summary is missing.

Research method: In this study, the PRISMA-driven systematic literature review is combined with the approach defined by Kitchenham (2004).

Main findings: The most frequently used data mining methods for defect detection are Bayesian network (BN) and Support vector machine (SVM). Besides previously mentioned methods, the Decision trees (DT) and Clustering are often used for defect classification. Neural Networks (NN) use is common for both defect detection and classification. DT, together with the Genetic algorithm (GA) and SVM, achieved the highest average accuracy. Recently, authors often combine different DM methods, and also methods for data dimensionality reduction are often used.

Implications for theory and practice: This study contributes to the quality management literature by extending a summary of recently used DM methods for defect detection and classification. This summary can help researchers choose a suitable method and build models for achieving its research purpose.

Keywords

Data mining. Defect. Detection. Classification. Manufacturing.

How to cite this article: Bártová, B., Bína, V., \& Váchová, L. (2022). A PRISMA-driven systematic review of data mining methods used for defects detection and classification in the manufacturing industry. Production, 32, e20210097. https:// doi.org/10.1590/0103-6513.20210097

Received: Aug. 3, 2021; Accepted: Dec. 8, 2021.

\section{Introduction}

Due to the rapid technological advancements and globalization the market and industrial environment is getting more complex, challenging and turbulent. To stay competitive firms need to fight for customers by research and inventions mainly in technological field. This gave rise to, in recent years frequently discussed phenomenon, Industry 4.0. which integrates the cyber-world with the physical systems by using semantic machine-to-machine communication, loT, CPS and embedded systems. Some authors, on the other hand, perceive that much of the shift towards manufacturing paradigm Industry 4.0 has been driven by the emergence of "Big-Data" and the issues associated with their collection, managing and interpretation which are still partially persist (Chen et al., 2015). To deal with the complexities of the modern production system within cyber-physical environment traditional manufacturing companies becomes so-called "smart factories" 
(Xu et al., 2018). The "smart" aspect involves the application of large amount of data gathered from sensors located on the production lines towards improvement of industrial processes, with the data actively being used to effect changes in a process, creating an intelligent feedback system (Wang et al., 2015). The big data enables to manufacturers to understand customers' needs in a holistic or all-encompassing manner and thanks to this almost all customers' needs could be mapped and analysed (Jiju, 2020). Closely related to the Industry 4.0 term, the field of quality management has also registered a raising trend regarding the possibility of using modern technologies to help increase performance, efficiency and product quality. Product quality is significant (and thus closely watched) factor for all types of companies' customers. It determines customer satisfaction and, together with other factors (e.g., price or promotion), directly influences the companies' revenues and market share. Like so, the term "Quality 4.0" has emerged, to help describe the relationship between quality management and Industry 4.0 practices (Raluca, 2021). The term "Quality 4.0" framed by Dan Jacob (Jacob, 2017) is interpreted as an integral part of Industry 4.0 and could be defined as the digitalization of TQM and its impact on quality technology, processes and people. Bowers \& Pickerel (2019) stated more specifically that Quality 4.0 is application of digitalization, artificial intelligence and so data mining methods to quality management. As Fayyad \& Stolorz (1997) stated already at the turn of this millennium, conventional statistical data analysis methods are no longer the best alternative to be used and this is all the more true for big data processing. A lot of applications contributing to support business decisions have already been promoted since the concepts of data mining were proposed in early 1990 (Fayyad et al., 1996). The huge amount of data in manufacturing databases contains large records with dozens of attributes that need to be explored simultaneously to discover useful information and knowledge, making manual analysis impractical. A detailed review of data mining research in manufacturing has been introduced by Harding et al. (2006) which together with Choudhary et al. (2009) are are considered to be the basic sources in this area. More recently Oliff \& Liu (2017) examined how data-mining principles and methods may be used for exploring the concept of Intelligent Manufacturing under lndustry 4.0 with a focus on improving product and process quality.

This paper aims to extend (not only) above mentioned reviews regarding the topic of Data mining methods application in manufacturing. Attention is paid especially to two parts of quality management in manufacturing, namely defect detection and defect classification in the manufacturing process. Different authors use different approaches, data mining methods and even some of them create new unique ones. In our future research we aim to create a new data mining approach as well and that is why the information, which methods are frequently and successfully used and basically, what has been already done within this topic, is a crucial issue for us.

\section{Background}

"Data Mining," often also referred to as "Knowledge Discovery in Databases" (KDD), is a relatively young sub-discipline of computer science aiming at the automatic interpretation of large datasets. The classic definition of knowledge discovery by Fayyad et al. from 1996 describes KDD as "[...] the non-trivial process of identifying valid, novel, potentially useful, and ultimately understandable patterns in data" (Fayyad et al. 1996, p. 41). The machine learning algorithms construct possible associations among various parameters (called features in data mining and attributes in computer database literature) that are important in modeling processes (Kusiak, 2001). The field of DM makes use of automated tools and techniques, employing sophisticated algorithms to discover hidden patterns, associations, anomalies, or structures from large amounts of data stored in a data warehouse or other information repositories, effective and valuable. In the context of manufacturing, two high-level primary goals of data mining can be identified - they are prediction and description. Descriptive data mining is focused on discovering interesting patterns to characterize the current data. Predictive data mining is meant to assess the behavior of a model and determine future values of key variables based on existing information provided by available databases (Choudhary et al., 2009). One of the most challenging tasks in the whole KDD process is to choose the right data mining technique.

Several approaches to the categorization of Data mining algorithms are available. The approach used in this study identifies strongly with Patel \& Patel (2014), although, for our purpose, the methodology was slightly modified. The scheme in Figure 1 represents a description of our approach. Data mining techniques can be roughly categorized into four major groups, namely Classification, Clustering, Association, and Regression. Classification is a process of determining groups ("classes") of given objects based on their characteristics, where the semantics of these classes is known beforehand. Classification and prediction are two forms of data analysis that can be used to extract models describing important data classes. Classification methods predict the target 


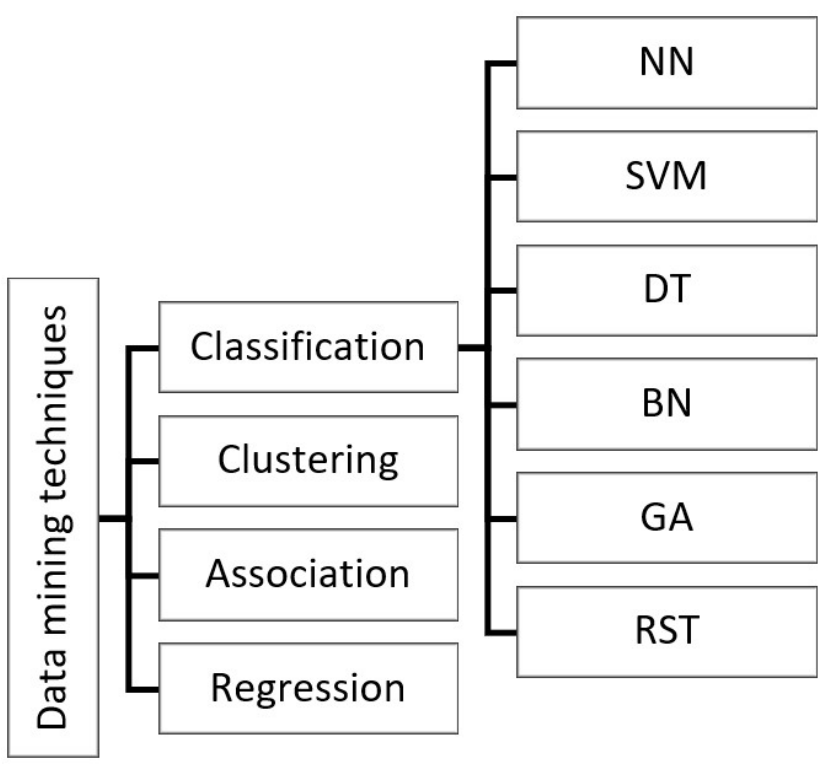

Figure 1. DM methods shceme.

class from analyzing the training dataset. To maximize predictive accuracy, the testing phase is then performed. In our work, this set of methods contains Neural networks (NN), Support vector machines (SVM), Decision trees (DT), Bayes networks (BN), Genetic algorithms (GA), and Rough set theory (RST). Clustering is the process of identifying natural groupings or clusters within multidimensional data based on some similarity measure. A brief characterization of the most popular DM tools, suitable also for manufacturing process analyses, can be found in Perzyk et al. (2007) or Perzyk (2006).

\section{Research method}

We have undertaken this survey in a systematic manner guided by the work of Kitchenham (2004), Kitchenham et al. (2010), and the PRISMA standards (Moher et al., 2009). Systematic literature review (SLR) was used to extract DM methods for production defect detection and classification and related questions. Based on the SLR and our research problem, the following research workflow (see Figure 2) can be raised. Through these research steps, we can obtain the desired results.

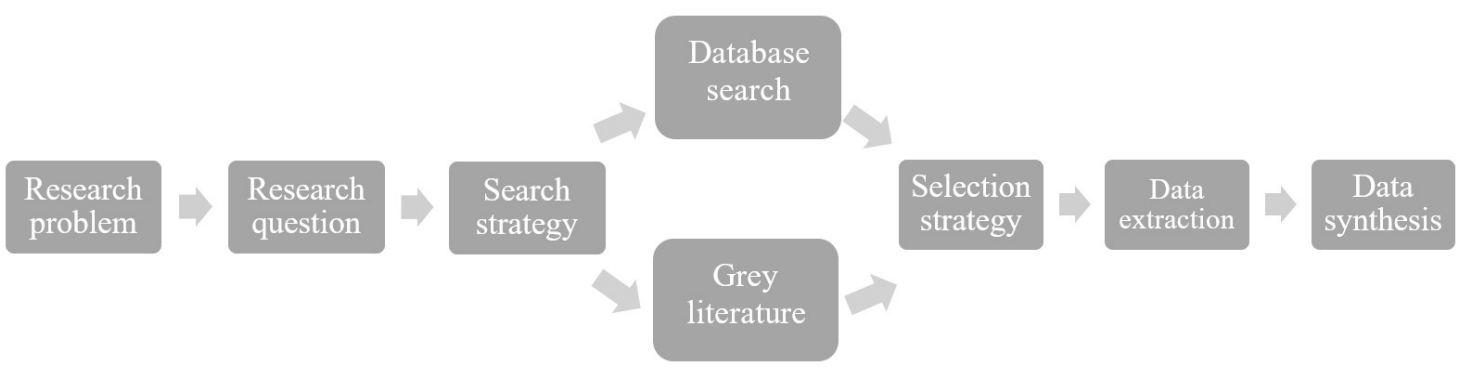

Figure 2. Research workflow.

\subsection{Research questions}

The primary research questions considered in this survey are mentioned in Table 1.

With respect to RQ1, we will search the relevant quality management literature employing data mining methods. 


\begin{tabular}{cl}
\hline Question ID & \multicolumn{1}{c}{ Research Question } \\
\hline RQ1 & Which are the most frequently used DM methods for defect detection and classification in the manufacturing process? \\
RQ2 & Which DM methods are the most efficient for defect classification/detection according to the chosen criteria? \\
RQ3 & Do authors combine different DM methods for quality management tasks in manufacturing? \\
RQ4 & Do authors use some dimensionality reduction methods for efficient work with high-volume data? \\
RQ5 & What are the main strength and limitations of individual DM methods? \\
\hline
\end{tabular}

\subsection{Search strategy}

\subsubsection{Stage 1: database search}

The first step is to define search keywords and validate the search string. We combined different keywords that are related to research questions. The list of used keywords is presented in Table 2.

The search string is defined as follows:

Table 2. Searched key words.

\begin{tabular}{cc}
\hline Item ID & Database Search Terms \\
\hline a & data mining \\
b & defect \\
c & detection \\
d & classification \\
e & data mining method \\
\hline
\end{tabular}

\section{ALl (a AND b AND (c OR d) AND e) in (Title or Abstract or Keyword)}

We used the ACM Digital library and ProQuest central as representative search engines to perform automatic searches and refine the search string until all the search items met the requirements and the number of remaining papers was minimal. Then, we used the defined search string to carry out automatic searches. We chose Scopus, ISI Web of Science, and ProQuest central (Table 3) because those three databases are the largest and most complete scientific databases, including high-quality papers regarding data mining problematic. After the automatic search, a total of 227067 papers were collected (Table 4).

Table 3. Study resources.

\begin{tabular}{cc}
\hline Source & Address \\
\hline Scopus & Scopus (2021) \\
1S1 Web of Science & Web of Science (2021) \\
ProQuest central & ProQuest (2021) \\
\hline
\end{tabular}

Table 4. Number of papers found in an automatic database search.

\begin{tabular}{lccc}
\hline \multicolumn{1}{c}{ Query } & \multicolumn{2}{c}{ Results } \\
\cline { 2 - 4 } & Scopus & WoS & ProQuest \\
\hline (“neural network”) AND (“defect”) AND (“detection) OR (“classification”) AND (“manufacturing”) & 498 & 605 & 23545 \\
(“support vector machine”) AND (“defect”) AND (“detection”) OR (“classification”) AND & 137 & 179 & 20574 \\
(“manufacturing”) & 5 & 5 & 4450 \\
(“baies network”) AND (“defect”) AND (“detection) OR (“classification”) AND (“manufacturing”) & 67 & 45 & 33910 \\
(“decision trees”) AND (“defect”) AND (“detection) OR (“classification”) AND (“manufacturing”) & 7 & 5 & 24605 \\
(“rough set theory”) AND (“defect”) AND (“detection) OR (“classification”) AND (“manufacturing”) & 48 & 53 & 15625 \\
(“genetic algorithm”) AND (“defect”) AND (“detection) OR (“classification”) AND (“manufacturing”) & 7 & 8 & 61043 \\
(“association”) AND (“defect”) AND (“detection) OR (“classification”) AND (“manufacturing”) & 84 & 179 & 17279 \\
(“clustering”) AND (“defect”) AND (“detection) OR (“classification”) AND (“manufacturing”) & 70 & 112 & 23922 \\
(“regression”) AND (“defect”) AND (“detection) OR (“classification”) AND (“manufacturing”) & &
\end{tabular}




\subsubsection{Stage 2: grey literature}

To cover gray literature, additional records were identified through other sources, f.e. Google Scholar, JSTOR, ACM, etc. Articles have been searched by keywords mentioned in Table 3. Some papers have been found during previous research (Bartova \& Bina, 2020). Detected articles were added to the papers pool from the database search.

The gathered articles are then filtered by using the selection strategy described in the next section.

\subsection{Selection strategy}

This methodological review was conducted in accordance with the Preferred reporting items for systematic reviews and meta-analyses (PRISMA) statement for searching, screening, appraising quality criteria, and data extraction and handling. This approach is used to describe employed research designs, methods, and procedures in the defect detection and classification process in manufacturing. It is used to highlight the strengths and weaknesses of methodological tools and explores the evaluation criteria for the performance and efficiency of used approaches. The review focuses on new methodological approaches, modifications of existing methods, and discussions of quantitative and data analytic approaches.

The original sample for this study included all journal articles published after 2010 found in the defined database by searching with defined keywords and key phrases. Using this searching strategy, 227067 articles were selected since they represent the most of researched knowledge. The set was then adjusted by applying the inclusion criteria. Because of the selected searching strategy, the set of articles tends to contain a large number of duplicities. The duplicities will be removed in the next step.

\subsubsection{Inclusion criteria}

- Journal articles and conference papers only

- Papers from January 2010 to March 2021

- Papers investigating defect detection or classification in the manufacturing process using some of Data mining methods

- Papers reported in the English language from any country

A total of 28255 articles met the inclusion criteria. After the removal of duplicities, we got the total number of 10877 papers which will be subject to the screening process itself. In the next part of the screening process, the 4-round exclusion process is used to reduce the source set of papers. The reduction process of the original set of papers is demonstrated in the PRISMA flow diagram (see Figure 3).

\subsubsection{Exclusion criteria}

\subsubsection{1. $1^{\text {st }}$ round exclusion criteria}

- Papers reported in non-English language from any country

- Systematic reviews, guidelines, organizational reports, abstract booklets, mathematical modeling studies

- Papers that are focused on non-manufacturing applications - healthcare, services, education, construction, biochemical industry

\subsubsection{2. $2^{\text {nd }}$ round exclusion criteria}

- Papers that are not empirical researches

- Papers focused on data quality

- Papers focused on maintenance, product design, assembling, inventory, etc. 


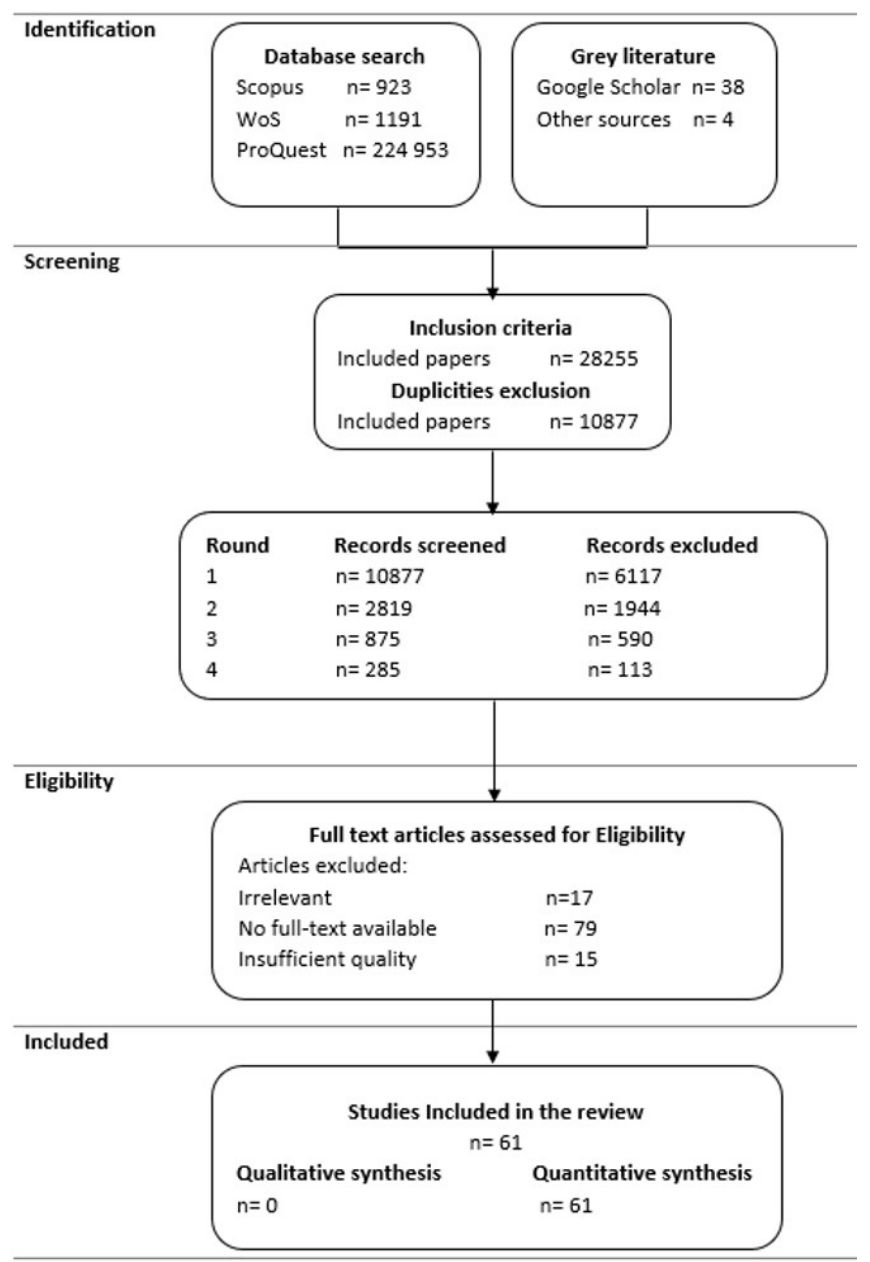

Figure 3. The process of primary study selection.

\subsubsection{3. $3^{\text {rd }}$ round exclusion criteria}

- Papers focused on fault detection

- Papers applying other methods than DM

- Papers focused on defects prediction

\subsubsection{4. $4^{\text {th }}$ round exclusion criteria}

- Papers that do not report primary data

- Papers focused on image processing, vibration signals, etc.

After the screening phase, 172 remaining articles are subjected to further analysis within the Eligibility phase. The abstracts of all articles are analyzed, and 17 articles are found to be irrelevant.

In addition to general inclusion/exclusion criteria, we consider it critical to assess the "quality" of primary studies. Since the full text of all articles needs to be provided, a total of 79 articles are removed from the research because of the impossibility of obtaining them. 


\subsection{Quality assessment}

In the quality assessment phase, the articles are evaluated according to the different points of view. The crucial aspects are clarity in research aims, methodology, and also results in description and discussion. The criteria for the quality of the study were determined according to the guidelines proposed by Kitchenham \& Charters (2007). The corresponding quality checklist is shown in Table 5. The table includes 12 questions that consider four research quality dimensions, including research design, conduct, analysis, and conclusions. For each quality item, we set a value of 1 if the authors put forward an explicit description, then a value of 0.5 if there was a vague description, and value 0 if there was no description at all. Each possible answer for each question has been scored in the main article and converted into a percentage.

Table 5. Quality assessment questions and results.

\begin{tabular}{|c|c|c|c|c|}
\hline \multirow{2}{*}{ Question ID } & \multirow{2}{*}{ Question } & \multicolumn{3}{|c|}{ Percentage } \\
\hline & & Yes & Partially & No \\
\hline \multicolumn{5}{|l|}{ Design } \\
\hline Q1 & Are the aims of the study clearly stated? & 68.85 & 29.51 & 1.64 \\
\hline Q2 & Are the chosen quality attributes distinctly defined? & 55.74 & 24.59 & 19.67 \\
\hline Q3 & Are the used DM methods discussed? & 36.07 & 45.90 & 18.03 \\
\hline \multicolumn{5}{|l|}{ Conduct } \\
\hline Q4 & 1s the used approach clearly described? & 75.41 & 24.59 & 0.00 \\
\hline Q5 & Are the datasets completely described (source. size. and programming languages)? & 29.51 & 29.51 & 40.98 \\
\hline Q6 & 1s it clear how accuracy was measured? & 57.38 & 11.48 & 31.15 \\
\hline \multicolumn{5}{|l|}{ Analysis } \\
\hline Q7 & Is the purpose of the analysis clear? & 73.77 & 26.23 & 0.00 \\
\hline Q8 & Were all model construction methods fully defined (use of tools and methods)? & 44.26 & 50.82 & 4.92 \\
\hline Q9 & Is the accuracy of the results reported? & 98.36 & 1.64 & 0.00 \\
\hline \multicolumn{5}{|l|}{ Conclusion } \\
\hline Q10 & Are the results compared with other methods? & 60.66 & 14.75 & 24.59 \\
\hline Q11 & Do the results support the conclusions? & 62.30 & 37.70 & 0.00 \\
\hline Q12 & Are validity threats discussed? & 21.31 & 29.51 & 49.18 \\
\hline
\end{tabular}

Based on the evaluated quality, we have decided to remove from our further research articles achieving less than 60\% quality. By this additional criterion, we reduced a total of 15 studies. These papers mainly had insufficiently described methodological approaches or results, which is unacceptable for fulfilling our research objectives.

A total of 61 articles were left after the quality assessment step. In general, only good-quality articles have been chosen. However, some of them do not describe the used dataset and accuracy measurements, and there is a lack of discussion of the limitations of the proposed method. However, the total average score of 8.47 out of 12 indicates that the quality of the research is promising, supporting the validity of the extracted data and the conclusions drawn therefrom.

\subsection{Data extraction}

The goal of this step is to design forms to identify and collect useful and relevant information from the selected primary studies so that they can answer our research questions proposed in Section 3.1. Table 6 shows the data extraction form, which will be applied to all selected primary studies for the purpose of detailed analysis.

\subsection{Data synthesis}

The search for our systematic review was performed in March 2021. Data synthesis is used to collect and summarize the data extracted from primary studies. Moreover, the main goal is to understand, analyze and extract frequently used DM methods for defect detection and classification applications in manufacturing processes. We analyzed the extracted data to determine the trends and collect information about our research questions. In addition, we classified and analyzed articles according to the research questions proposed in Section 3.1. We also classified the literature according to different research questions. The most important task was to classify the articles according to the DM methods through the relevant analysis. 


\begin{tabular}{cllc}
\hline \multicolumn{1}{c}{ Extracted data } & \multicolumn{1}{c}{ Description } & Type \\
\hline 1 & Authors & Research author's other relevant studies & Whole research \\
2 & Publication year & Indicating the trend of research & Whole research \\
3 & Study title & Reflect the relevant research direction & Whole research \\
4 & Context study & Understand the full text & Whole research \\
5 & DM method or combinations & Methods used in the model & RQ1, RQ2 \\
6 & Achieved accuracy & The effectiveness of the methods & RQ2, RQ3 \\
7 & Method strengths & The advantages of the approach & RQ5 \\
8 & Method limitation & The disadvantages of the approach & RQ5 \\
9 & Dimensionality reduction & Method used for dimensionality reduction & RQ4 \\
\hline
\end{tabular}

\section{Results}

In this section, we will deeply analyze the primary studies to provide an answer to the five research questions presented in Section 3. While Section 4.1 provides an overview of the main concepts discussed in this section, Sections 4.2-4.6 report the answer to the research questions.

\subsection{Overview of the DM methods}

First, let us consider the question of frequency in using data mining methods for defect detection and their classification in the manufacturing industry. In order to achieve it, the particular methods were basically mined from the final primary studies base. As you can see on the pie chart below (Figure 4) classification technique is conclusively the most frequently used (88.5\% from all included articles). That is why we compared the classification algorithms separately. The Neural network (70.37\%) and Support vector machine (16.67\%) were identified as the most frequently used. Decision trees rank within the list of frequently used methods as well, with a share bigger than 5.56\%. The particular DM method is often chosen with respect to the nature of the data and according to the information type intended to be mined. As Han \& Kamber (2001) mentioned - the kind of knowledge to be mined determines the data mining functions to be performed. This statement is also supported by Gibert et al. (2010), who believe that the choice of the DM method used depends on both goals of the problem to be solved and the structure of the available data set.

\section{Data mining methods}

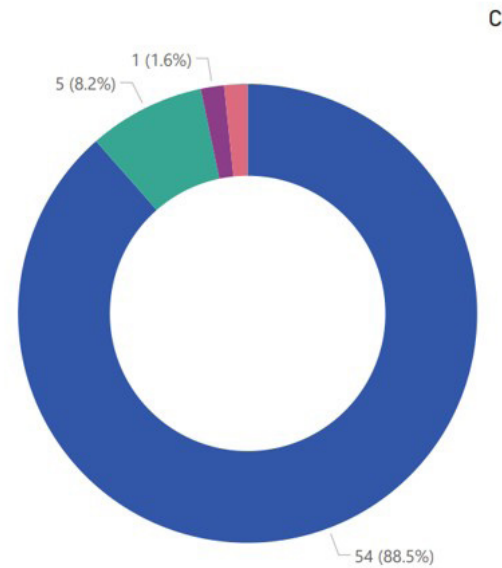

Classification techniques

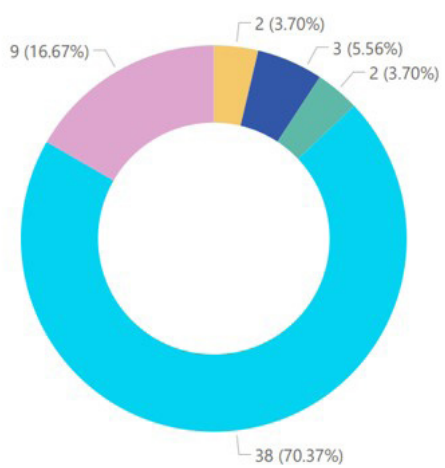

Figure 4. DM methods usage chart.

The number of papers from our set of articles distributed over the years is shown in Figure 5. We can see that from 2017 the number of published articles focused on DM methods application in defect detection and classification has significantly increased. So far, the highest number of studies was published in 2020, but the number of studies for 2021 is taken only for the first quarter. From this, we can assume that our research topic is currently often discussed among researchers from around the world. Figure 6 then shows the particular DM 


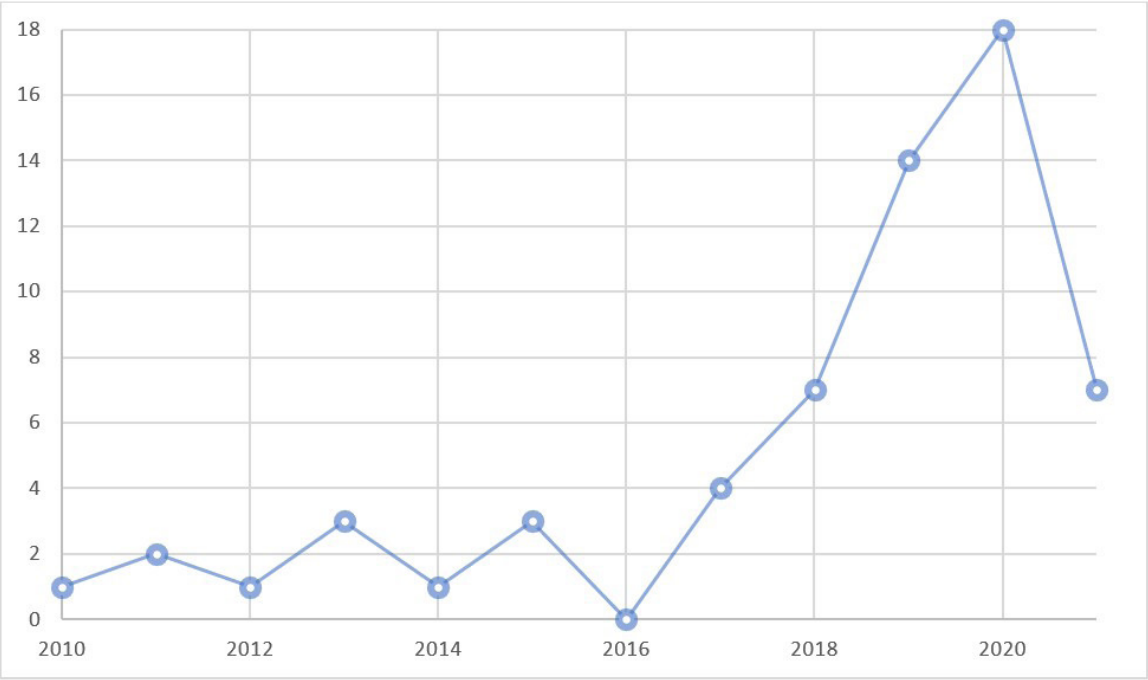

Figure 5. Distribution of papers over the years 2010-2021.

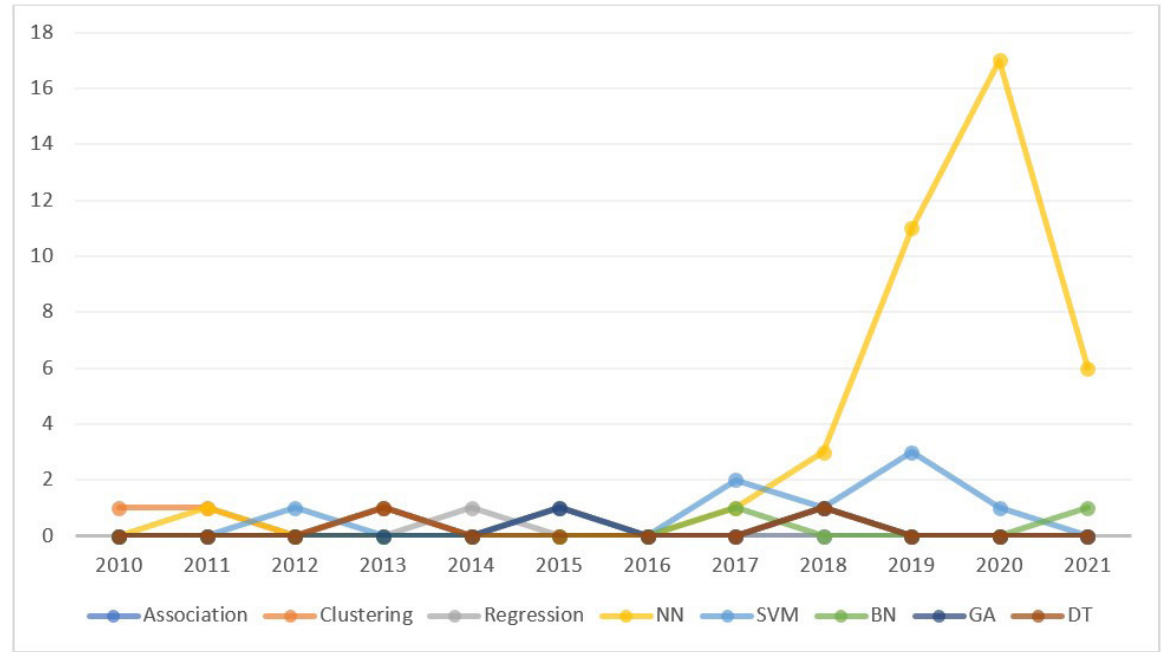

Figure 6. DM methods usability over the years 2010-2021.

methods usability distributed over the years. We can see that the Neural network method is the most frequently used, especially in recent years (total of 17 studies in 2020). There is also growth for the SVM method from the year 2017 (with 2 applications in 2017 and 3 in 2019). From the chart, we can also assume that BN method usability will increase in the near future. On the other hand, Regression and Clustering methods are probably already behind the zenith and are no more perspectives for us.

Now, let us look more closely at the considered DM methods in connection with analyzed papers.

- Association rules

Generally, association rules are not frequently used for defect detection and classification in the manufacturing industry. They are usually employed for the search of relations among individual items (for example, in the shopping basket). Despite that, one paper related to hybrid OLAP-association rule mining based quality management system for extracting defect patterns in the garment industry (Lee et al., 2013) was found in our article base.

- Regression 
Similarly, the use of regression analysis for defect detection and their classification is quite rare. The main application area for regression analysis can be identified in the field of defect prediction. Our research detected the only study of defects detection in gearbox where Spectral regression (SR) is combined with the SVM method (Jiang et al., 2014).

\section{- Genetic algorithm}

Also, Genetic algorithms were not often used. We have only 2 cases in our final set of articles. Apostolos Chondronasios et al. (2016) used this method for feature selection purposes within the surface defect classification task. Later, Ji-Deok et al. (2018) proposed a combination of genetic algorithms and support vector machines for defect classification in the PCB manufacturing process, which achieved 96\% accuracy.

\section{- Neural Networks}

Regarding our selected set of papers, the most frequently used and probably the most successful data mining method for defect product detection or classification is the Neural network. Most recently, for example, the article "Unsupervised Pre-Training of Imbalanced Data for Identification of Wafer Map Defect Patterns" written by Shon et al. (2021), where the proposed model is based on convolution variational autoencoder (CVAE), achieved a $95.1 \%$ level of F-measure. In our analysis, we found out that more than $42 \%$ of composed primary studies implementing NN are focused on CNN, for example, Lin et al. (2019), Wang et al. (2018), Imoto et al. (2019), Lee et al. (2019), Lee \& Lee (2019), Yang et al. (2019), Chen et al. (2020a), Chien et al. (2020), Han \& Yu (2020), Lee \& Ryu (2020), Batool et al. (2020), Lin et al. (2020), Yang et al. (2020), Xu et al. (2020), Cerezci et al. (2020) or Jiang et al. (2021). R-CNN algorithm is also often used (more than 13\%), for example, in Tabernik et al. (2019), Shi et al. (2020a), Liyun et al. (2020), Zhao et al. (2020a), Zhang \& Shen (2021) or Zhao et al. (2021). Moreover, there are articles focusing on the implementation of Contextual Hopfield Neural Network (Chang et al., 2011), Sparse Convolutional Neural Networks (Bella et al., 2019), FCN (Zhang et al., 2019), ResNet50 (Konovalenko et al., 2020), VGG16 (lhar et al., 2019), YOLOv3 (Yu et al., 2019). The rest of the studies focused on NN can be seen in Table 7.

\section{- Decision trees}

In our set of papers, decision trees are successfully used mainly for defect classification in manufacturing processes. For example, Radhika et al. (2013) proposed a model for defect pattern recognition based on random forest. We can also mention a study from Mao et al. (2018), "An Improved Skewness Decision Tree SVM Algorithm for the Classification of Steel Cord Conveyor Belt Defects," where authors combine decision tree with SVM with total accuracy of $97 \%$.

\section{- Clustering}

One of the most often used data mining methods for defect detection and classification in the manufacturing process is clustering. For example, Chia-Yu Hsu (2015) proposed a clustering algorithm for defect classification in semiconductor manufacturing. Also, Taha et al. (2018) focused on the semiconductor industry, where successfully performed a clustering algorithm for defect pattern recognition. It is also worth mentioning the article "Bayesian spatial defect pattern recognition in semiconductor fabrication using support vector clustering" despite Yuan et al. wrote it in 2010. This study combines different algorithms with total accuracy of up to $96.55 \%$, and for this reason, it is interesting and beneficial for our research.

\section{- Support vector machine}

After NN, the Support vector machine algorithm is the second most frequently used method in our primary set of articles. Prachya Bumrungkun (2019) used the SVM method for defect detection in the textile manufacturing industry. In the same year, Li et al. (2019) also used SVM but for surface defects detection in Polycrystalline Diamond Compact manufacturing. Takada et al. (2017) successfully combined SVM with CNN for Electronic Circuit Boards defect detection and classification with an accuracy of 82.64\%. Liu et al. (2019) achieved an accuracy of 99.95\% by its SVM model for the real-time defect detection task. Also, Baly \& Hajj (2012) and Jeong (2017) have achieved good results with the SVM method implementation in semiconductor manufacturing. Lastly, we would like to mention the study from Hoang \& Nguyen (2020), where the authors proposed a sequence of SVM and state-of-the-art history-based adaptive differential evolution with linear population size reduction for concrete quality management, which achieved an accuracy of $93 \%$. 


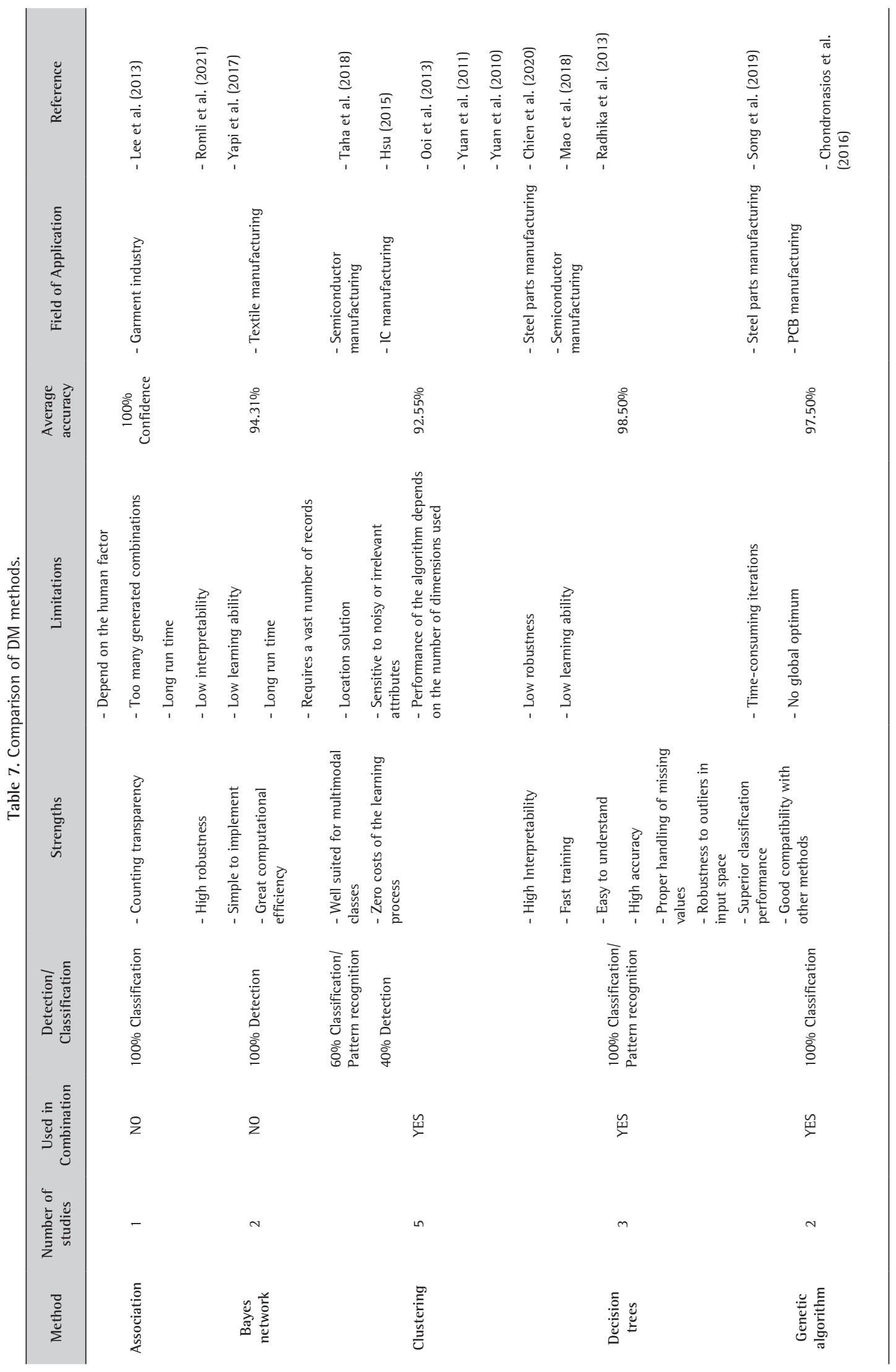




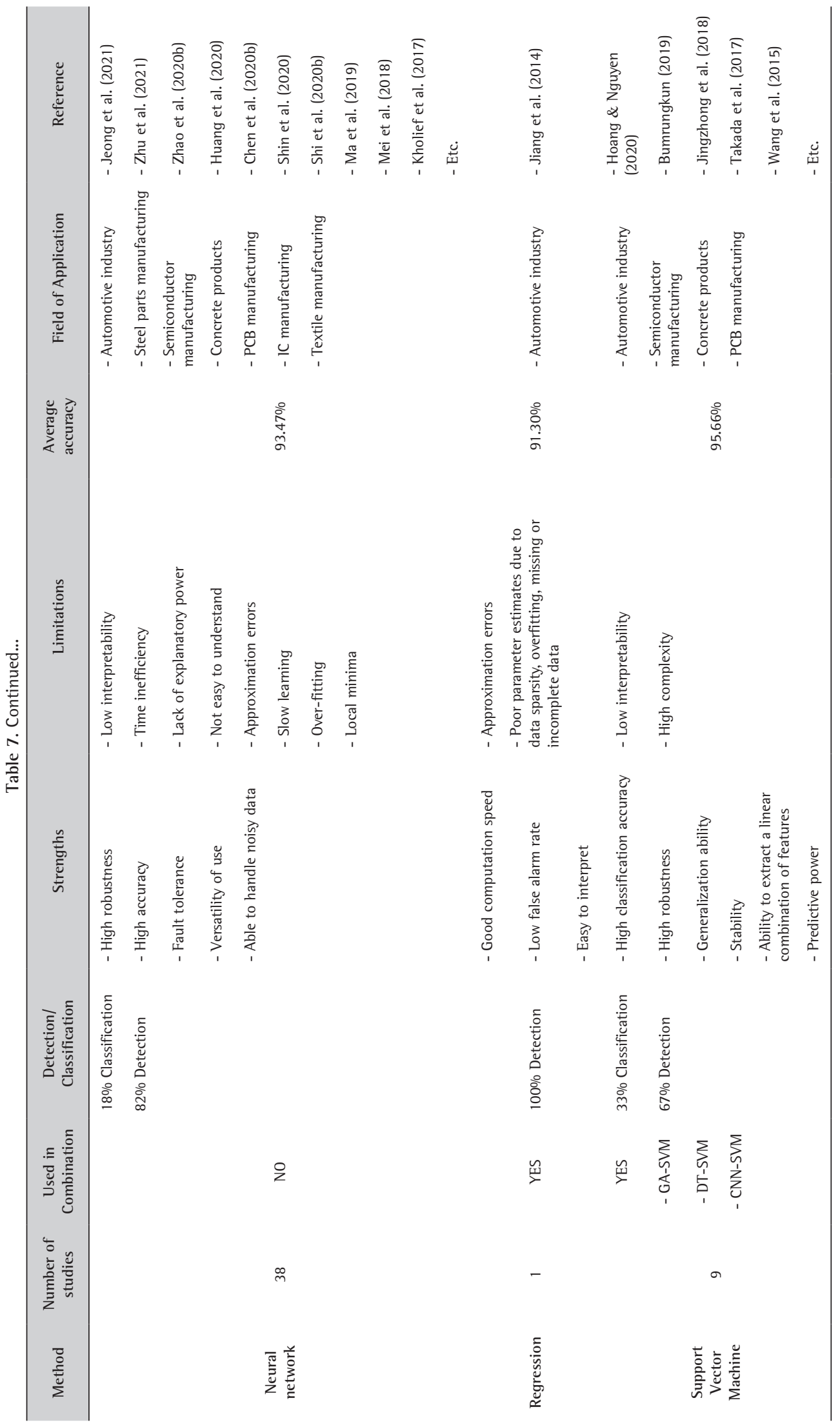


In the following table (Table 7), we summarize individual DM methods, which were found out in our analysis as the most frequently used for defect detection and classification in the manufacturing industry and their average accuracy. The main message of this table is the strengths and limitations of these methods, their frequent field of application, and information on whether the particular method is used in combination with other methods or not. This table will help us in choosing methods for our future research.

\subsection{Discussion of research questions}

- RQ1: Which are the most frequently used DM methods for defect detection and classification in the manufacturing process?

The goal of this section is to answer the RQ1 (which mentioned DM methods are the most frequently used for defect detection and classification tasks?). Overall, the most commonly used method and also the most popular method in recent years is the neural network. There are several different algorithms used, such as CNN, R-CNN, FCN, ResNet50, etc. The NN method is mainly used for defect detection as well as SVM. For defect classification problems, the Clustering method and Decision trees are the most frequently used. It is necessary to mention that NN, SVM, and Clustering are often used for either defect detection or classification.

- RQ2: Which DM methods are the most efficient for defect classification/detection according to the chosen criteria?

The DM method efficiency can be measured, for example, by F-measure, AOC, or simple accuracy. We have compared the particular DM methods by average accuracy achieved by the proposed method. As you can see from Figure 7, the highest average accuracy was achieved by Decision trees, Genetic algorithms, and SVM methods. During the analysis of individual primary studies, we observed that NN, as well as SVM, have achieved in some cases even 100\% accuracy, but due to the high volume of studies implementing in the case of NN, the final result can be distorted. For this purpose, we decided to add a median that is less sensitive to extreme values. So from the median point of view, we can see that accuracy of NN, SVM, and Clustering methods is significantly higher.

- RQ3: Do authors combine different DM methods for quality management tasks in manufacturing?

Recently it has become more common for authors to try to combine different data mining methods and use them parallelly or sequentially. In our primary set of articles, we deduced that approximately $30 \%$ of gathered

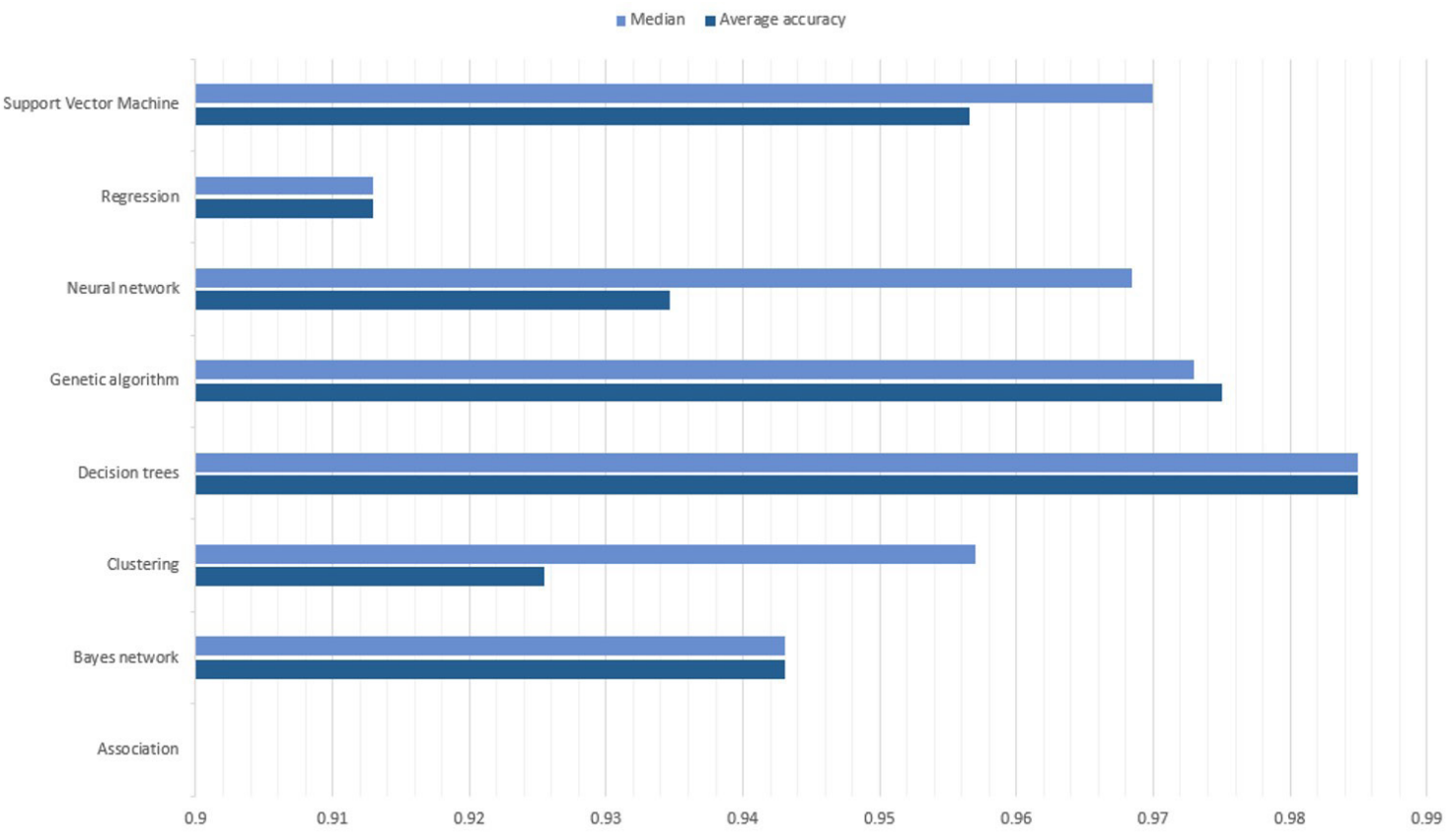

Figure 7. DM methods average and median accuracy. 
articles use more than one DM method. We noticed that SVM is often combined with other methods, such as Genetic algorithms, Decision trees, or Neural networks. On the other hand, we can say that generally, the NN method is not used in combination with other different methods, but sometimes various NN types are combined together.

- RQ4: Do authors use some dimensionality reduction methods for efficient work with high volume data?

Generally yes, several studies in our research use some dimensionality reduction methods, such as Principal Component Analysis, Random forest, Factor analysis, or ReliefF algorithm. The dimensionality reduction method was detected in more than 15\% of studies, but it is possible that it is not always mentioned in the study methodology or it is not clear.

- RQ5: What are the main strength and limitations of individual DM methods?

The complete list of strengths and limitations of particular methods is contained in Table 7, but we can at least discuss here a few of them. Surprisingly, NN, which is the most frequently used, has quite a long list of weaknesses such as low interpretability, time inefficiency, or over-fitting. In contrast, SVM appears to be more effective. Among its strengths, we can mention, for example, high robustness, generalization ability, and high accuracy.

\section{Conclusion}

This paper introduced a PRISMA-based systematic review focused on defect detection and classification in the manufacturing process using data mining methods. 28255 high-quality articles in the SCOPUS, WoS, and ProQuest Central databases were searched out from who's the final set of only 61 empirical researches were extracted by sequential selection. It was found out that the most frequently used DM methods for the topic under consideration are NN, SVM, Clustering, and DT. Most importantly, we found out that DT, GA, and SVM achieve the highest average accuracy. The advantages and disadvantages of particular data mining methods based on analyzed articles were identified as well. Additionally, from our research results,, we can assume that researchers use different DM methods in combinations and use some data dimensionality reduction methods such as PCA or Random forest. We also found out that the SVM and BN approaches have significant advantages and seem suitable for defect detection tasks. On the other hand, the application of Neural networks brings a lot of disadvantages, which makes them more complicated to be used, but despite this, they are often used for both defect detection and classification. The clustering method is also quite often used mainly for defect classification and achieves good results. It is necessary to state that the results presented in this paper come with certain limitations, especially with respect to the use of three selected source databases to obtain the original set of papers for analysis. We are also aware of some limits in accuracy assessment by average and median values.

This research helps us to design our own model for defect detection and classification, which will be our main interest in future work.

\section{References}

Baly, R., \& Hajj, H. (2012). Wafer classification using support vector machines. IEEE Transactions on Semiconductor Manufacturing, 25(3), 373-383. http://dx.doi.org/10.1109/TSM.2012.2196058.

Bartova, B., \& Bina, V. (2020). Data mining methods used for quality management: a bibliometric analysis. In The 4th International Conference on Digital Technology in Education. New York: Association for Computing Machinery. http://dx.doi.org/10.1145/3429630.3429646

Batool, U., Shapiai, M. 1., Fauzi, H., \& Fong, J. X. (2020). Convolutional neural network for imbalanced data classification of silicon wafer defects. In 16th IEEE International Colloquium on Signal Processing \& its Applications. USA: IEEE. http://dx.doi.org/10.1109/ CSPA48992.2020.9068669

Bella, R., Carrera, D., Rossi, B., Fragneto, P., \& Boracchi, G. (2019). Wafer defect map classification using sparse convolutional networks. In E. Ricci, S. R. Bulò, C. Snoek, O. Lanz, S. Messelodi \& N. Sebe (Eds.), International Conference on Image Analysis and Processing (pp. 125-136). Trento, ltaly: Springer. http://dx.doi.org/10.1007/978-3-030-30645-8_12.

Bowers, K., \& Pickerel, T. V. (2019). Vox Populi 4.0: big data tools zoom in on the voice of the customer. Quality Progress, 52(3), 32-39.

Bumrungkun, P. (2019). Defect detection in textile fabrics with snake active contour and support vector machines. Journal of Physics: Conference Series, 1195, 012006. http://dx.doi.org/10.1088/1742-6596/1195/1/012006.

Cerezci, F., Kazan, S., Oz, M. A., Oz, C., Tasci, T., Hizal, S., \& Altay, Ç. (2020). Online metallic surface defect detection using deep learning. Emerging Materials Research, 9(4), 1266-1273. http://dx.doi.org/10.1680/jemmr.20.00197.

Chang, C., Li, C., Chang, Y., \& Jeng, M. (2011). Wafer defect inspection by neural analysis of region features. Journal of Intelligent Manufacturing, 22(6), 953-964. http://dx.doi.org/10.1007/s10845-009-0369-4. 
Chen, F., Deng, P., Wan, J., Zhang, D., Vasilakos, A. V., \& Rong, X. (2015). Data mining for the internet of things: literature review and challenges. International Journal of Distributed Sensor Networks, 11(8), 431047. http://dx.doi.org/10.1155/2015/431047.

Chen, H., Pang, Y., Hu, Q., \& Liu, K. (2020a). Solar cell surface defect inspection based on multispectral convolutional neural network. Journal of Intelligent Manufacturing, 31(2), 453-468. http://dx.doi.org/10.1007/s10845-018-1458-z.

Chen, X., Chen, J., Han, X., Zhao, C., Zhang, D., Zhu, K., \& Su, Y. (2020b). A light-weighted CNN model for wafer structural defect detection. IEEE Access: Practical Innovations, Open Solutions, 8, 24006-24018. http://dx.doi.org/10.1109/ACCESS.2020.2970461.

Chien, J., Wu, M., \& Lee, J. (2020). Inspection and classification of semiconductor wafer surface defects using CNN deep learning networks. Applied Sciences, 10(15), 5340. http://dx.doi.org/10.3390/app10155340.

Chondronasios, A., Popov, 1., \& Jordanov, 1. (2016). Feature selection for surface defect classification of extruded aluminum profiles. International Journal of Advanced Manufacturing Technology, 83(1-4), 33-41. http://dx.doi.org/10.1007/s00170-015-7514-3.

Choudhary, A. K., Harding, J., \& Tiwari, M. K. (2009). Data mining in manufacturing: a review based on the kind of knowledge. Journal of Intelligent Manufacturing, 20(5), 501-521. http://dx.doi.org/10.1007/s10845-008-0145-x.

Fayyad, U. M., Piatetsky-Shapiro, G., \& Smyth, P. (1996). From data mining to knowledge discovery: an overview. In U. M. Fayyad, G. Piatetsky-Shapiro, P. Smyth \& R. Uthurusamy (Eds.), Advances in knowledge discovery and data mining. Menlo Park: AAAl Press, p.41

Fayyad, U., \& Stolorz, P. (1997). Data mining and KDD: promise and challenges. Future Generation Computer Systems, 13(2-3), 99-115. http://dx.doi.org/10.1016/S0167-739X(97)00015-0.

Gibert, K., Sànchez-Marrè, M., \& Codina, V. (2010). Choosing the right data mining technique: classification of methods and intelligent recommendation. In International Environmental Modelling and Software Society Vol. 5. Ontario, Canadá: iEMSs.

Han, J., \& Kamber, M. (2001). Data mining: concepts and techniques. Academic Press, San Diego

Han, Y., \& Yu, H. (2020). Fabric defect detection system using stacked convolutional denoising auto-encoders trained with synthetic defect data. Applied Sciences, 10(7), 2511. http://dx.doi.org/10.3390/app10072511.

Harding, J. A., Shahbaz, M., Srinivas, \& Kusiak, A. (2006). Data mining in manufacturing: a review. Journal of Manufacturing Science and Engineering, 128(4), 969-976. http://dx.doi.org/10.1115/1.2194554.

Hoang, N., \& Nguyen, Q. (2020). A novel approach for automatic detection of concrete surface voids using image texture analysis and history-based adaptive differential evolution optimized support vector machine. Advances in Civil Engineering, 2020, 1-15. http:// dx.doi.org/10.1155/2020/4190682.

Hsu, C.-Y. (2015). Clustering ensemble for identifying defective wafer bin map in semiconductor manufacturing. Mathematical Problems in Engineering, 2015, 1-11. http://dx.doi.org/10.1155/2015/707358.

Huang, Y., Qiu, C., Wang, X., Wang, S., \& Yuan, K. (2020). A compact convolutional neural network for surface defect inspection. Sensors, 20(7), 1974. http://dx.doi.org/10.3390/s20071974. PMid:32244764.

Ihar, V., Mujeeb, A., Wenting, D., Marius, E., \& Alexei, S. (2019). Detection defect in printed circuit boards using unsupervised feature extraction upon transfer learning. In 2019 International Conference on Cyberworlds (pp. 101-108). USA: IEEE.

Imoto, K., Nakai, T., Ike, T., Haruki, K., \& Sato, Y. (2019). A CNN-based transfer learning method for defect classification in semiconductor manufacturing. IEEE Transactions on Semiconductor Manufacturing, 32(4), 455-459. http://dx.doi.org/10.1109/TSM.2019.2941752.

Jacob, D. (2017). Quality 4.0 impact and strategy handbook: getting digitally connected quality management. Retrieved in 2021, May 24, from https://generisgp.com/2018/02/15/the-quality-4-0-impact-and-strategy-handbook/.

Jeong, E., Kim, J., Jang, W., Lim, H., Noh, H., \& Choi, J. (2021). A more reliable defect detection and performance improvement method for panel inspection based on artificial intelligence. Journal of Information Display, 22(3), 127-136. http://dx.doi.org/10.1080/15 980316.2021.1876174.

Jeong, Y. (2017). Semiconductor wafer defect classification using support vector machine with weighted dynamic time warping kernel function. Industrial Engineering \& Management Systems, 16(3), 420-426. http://dx.doi.org/10.7232/iems.2017.16.3.420.

Jiang, H., Hu, Q., Zhi, Z., Gao, J., Gao, Z., Wang, R., He, S., \& Li, H. (2021). Convolution neural network model with improved pooling strategy and feature selection for weld defect recognition. Welding in the World, 65(4), 731-744. http://dx.doi.org/10.1007/s40194020-01027-6.

Jiang, Y., Wu, J., \& Zong, C. (2014). An effective diagnosis method for single and multiple defects detection in gearbox based on nonlinear feature selection and kernel-based extreme learning machine. Journal of Vibroengineering, 16(1), 499-512.

Ji-Deok, S., Young-Gyu, K., \& Tae-Hyoung, P. (2018). SMT defect classification by feature extraction region optimization and machine learning. International Journal of Advanced Manufacturing Technology, 101(5-8), 1303-1313.

Jiju, A. (2020). Quality 4.0: taking quality to its next level. Industrial and Systems Engineering at Work, 52(6), 46-47. Retrieved in 2021, April 8, from https://www.proquest.com/docview/2411183836/abstract/CD8BFC5C54304FFEPQ/1 ?accountid=17203

Jingzhong, H., Kewen, X., Fan, Y., \& Baokai, Z. (2018). Strip steel surface defects recognition based on socp optimized multiple kernel RVM. Mathematical Problems in Engineering, 2018, 1-8. http://dx.doi.org/10.1155/2018/9298017.

Kholief, E. A., Darwish, S. H., \& Fors, M. N. (2017). Detection of steel surface defect based on machine learning using deep auto-encoder network. In Proceedings of the International Conference on Industrial Engineering and Operations Management. Rabat, Morocco, IE0M.

Kitchenham B. (2004). Procedure for undertaking systematic reviews: technical report. Keele; Eveleigh: Keele University; National ICT Australia Ltd.

Kitchenham, B., \& Charters, S. (2007). Guidelines for performing systematic literature reviews in software engineering. Keele; Durham: Keele University; University of Durham.

Kitchenham, B., Pretorius, R., Budgen, D., Brereton, O. P., Turner, M., Niazi, M., \& Linkman, S. (2010). Systematic literature reviews in software engineering: a tertiary study. Information and Software Technology, 52(8), 792-805. http://dx.doi.org/10.1016/j. infsof.2010.03.006.

Konovalenko, l., Maruschak, P., Brezinova, J., Viňáš, J., \& Brezina, J. (2020). Steel surface defect classification using deep residual neural network. Metals, 10(6), 846. http://dx.doi.org/10.3390/met10060846. 
Kusiak, A. (2001). Feature transformation methods in data mining. IEEE Transactions on Electronics Packaging Manufacturing, 24(3), 214-221. http://dx.doi.org/10.1109/6104.956807.

Lee, C. K. H., Choy, K. L., Ho, G. T. S., Chin, K. S., Law, K. M. Y., \& Tse, Y. K. (2013). A hybrid OLAP-association rule mining based quality management system for extracting defect patterns in the garment industry. Expert Systems with Applications, 40(7), 2435-2446. http://dx.doi.org/10.1016/j.eswa.2012.10.057.

Lee, H., \& Ryu, K. (2020). Dual-kernel-based aggregated residual network for surface defect inspection in injection molding processes. Applied Sciences, 10(22), 8171. http://dx.doi.org/10.3390/app10228171.

Lee, J., \& Lee, J. (2019). A reliable defect detection method for patterned wafer image using convolutional neural networks with the transfer learning. IOP Conference Series: Materials Science and Engineering, 647, 012010. http://dx.doi.org/10.1088/1757$899 X / 647 / 1 / 012010$

Lee, S. Y., Tama, B. A., Moon, S. J., \& Lee, S. (2019). Steel surface defect diagnostics using deep convolutional neural network and class activation map. Applied Sciences, 9(24), 5449. http://dx.doi.org/10.3390/app9245449.

Li, H., Cui, C., \& Guo, H. (2019). Identification and classification of surface defects in polycrystalline diamond compact. In International Symposium on Precision Mechanical Measurements, Vol. 11343. Chongqing, China: SPIE. http://dx.doi.org/10.1117/12.2547602.

Lin, H., Li, B., Wang, X., Shu, Y., \& Niu, S. (2019). Automated defect inspection of LED chip using deep convolutional neural network. Journal of Intelligent Manufacturing, 30(6), 2525-2534. http://dx.doi.org/10.1007/s10845-018-1415-x.

Lin, Z., Ye, H., Zhan, B., \& Huang, X. (2020). An efficient network for surface defect detection. Applied Sciences, 10(17), 6085. http:// dx.doi.org/10.3390/app10176085.

Liu, T., Huang, L., \& Chen, B. (2019). Real-time defect detection of laser additive manufacturing based on support vector machine. Journal of Physics: Conference Series, 1213(5), 052043. http://dx.doi.org/10.1088/1742-6596/1213/5/052043.

Liyun, X., Boyu, L., Hong, M., \& Xingzhong, L. (2020). Improved faster R-CNN algorithm for defect detection in powertrain assembly line. Procedia CIRP, 93, 479-484. http://dx.doi.org/10.1016/j.procir.2020.04.031.

Ma, L., Xie, W., \& Zhang, Y. (2019). Blister defect detection based on convolutional neural network for polymer lithium-ion battery. Applied Sciences, 9(6), 1085. http://dx.doi.org/10.3390/app9061085.

Mao, Q., Ma, H., Zhang, X., \& Zhang, G. (2018). An improved skewness decision tree svm algorithm for the classification of steel cord conveyor belt defects. Applied Sciences, 8(12), 2574. http://dx.doi.org/10.3390/app8122574.

Mei, S., Wang, Y., \& Wen, G. (2018). Automatic fabric defect detection with a multi-scale convolutional denoising autoencoder network model. Sensors, 18(4), 1064. http://dx.doi.org/10.3390/s18041064. PMid:29614813.

Moher, D., Liberati, A., Tetzlaff, J., \& Altman, D. (2009). Preferred reporting items for systematic reviews and meta-analyses: the prisma statement. Journal of Clinical Epidemiology, 62(10), 1006-1012. http://dx.doi.org/10.1016/j.jclinepi.2009.06.005. PMid:19631508.

Oliff, H., \& Liu, Y. (2017). Towards Industry 4.0 utilizing data-mining techniques: a case study on quality improvement. Procedia CIRP, 63, 167-172. http://dx.doi.org/10.1016/j.procir.2017.03.311.

Ooi, M., Sok, H. K., Kuang, Y. C., Demidenko, S., \& Chan, C. (2013). Defect cluster recognition system for fabricated semiconductor wafers. Engineering Applications of Artificial Intelligence, 26(3), 1029-1043. http://dx.doi.org/10.1016/j.engappai.2012.03.016.

Patel, H. P., \& Patel, D. (2014). A brief survey of data mining techniques applied to agricultural data. International Journal of Computers and Applications, 95(9), 6-8. http://dx.doi.org/10.5120/16620-6472.

Perzyk, M. (2006). Data mining in foundry production. In K. Świątkowski (Ed.), Research in Polish metallurgy at the beginning of XXI century (pp. 255-275). Poland: Committee of Metallurgy of the Polish Academy of Sciences.

Perzyk, M., Biernacki, R., \& Kozlowski, J. (2007). Data mining in manufacturing: methods, potentials, limitations. In Proceedings of the 2007 Advances in Production Engineering Conference (pp. 147-156). Poland: Akapit.

ProQuest. (2021). Retrieved in 14 March 2021, from https://www.proquest.com/.

Radhika, N., Senapathi, S. B., Subramaniam, R., Subramany, R., \& Vishnu, K. N. (2013). Pattern recognition based surface roughness prediction in turning hybrid metal matrix composite using random forest algorithm. Industrial Lubrication and Tribology, 65(5), 311-319. http://dx.doi.org/10.1108/lLT-02-2011-0015.

Raluca, D. (2021). Knowledge management systems in Quality 4.0. MATEC Web of Conferences, 342, 09003.

Romli, l., Pardamean, T., Butsianto, S., Wiyatno, T. N., \& Mohamad, E. (2021). Naive bayes algorithm implementation based on particle swarm optimization in analyzing the defect product. Journal of Physics: Conference Series, 1845(1), 012020. http://dx.doi. org/10.1088/1742-6596/1845/1/012020.

Scopus. (2021). Retrieved in 12 March 2021, from http://www.scopus.com/.

Shi, J., Li, Z., Zhu, T., Wang, D., \& Ni, C. (2020a). Defect detection of industry wood veneer based on NAS and multi-channel Mask R-CNN. Sensors, 2O(16), 4398. http://dx.doi.org/10.3390/s20164398. PMid:32781740.

Shi, W., Zhang, L., Li, Y., \& Liu, H. (2020b). Adversarial semi-supervised learning method for printed circuit board unknown defect detection. Journal of Engineering, 2020(13), 505-510. http://dx.doi.org/10.1049/joe.2019.1181.

Shin, S., Jin, C., Yu, J., \& Rhee, S. (2020). Real-time detection of weld defects for automated welding process base on deep neural network. Metals, 10(3), 389. http://dx.doi.org/10.3390/met10030389.

Shon, H. S., Batbaatar, E., Cho, W., \& Choi, S. G. (2021). Unsupervised pre-training of imbalanced data for identification of wafer map defect patterns. IEEE Access: Practical Innovations, Open Solutions, 9, 52352-52363. http://dx.doi.org/10.1109/ACCESS.2021.3068378.

Song, J., Kim, Y., \& Park, T. (2019). SMT defect classification by feature extraction region optimization and machine learning. International Journal of Advanced Manufacturing Technology, 101(5-8), 1303-1313. http://dx.doi.org/10.1007/s00170-018-3022-6.

Tabernik, D., Šela, S., Skvarč, J., \& Skočaj, D. (2019). Segmentation-based deep-learning approach for surface-defect detection. Journal of Intelligent Manufacturing, 31(3), 759-776. http://dx.doi.org/10.1007/s10845-019-01476-x.

Taha, K., Salah, K., \& Yoo, P. D. (2018). Clustering the dominant defective patterns in semiconductor wafer maps. IEEE Transactions on Semiconductor Manufacturing, 31(1), 156-165. http://dx.doi.org/10.1109/TSM.2017.2768323. 
Takada, Y., Shiina, T., Usami, H., Iwahori, Y., \& Bhuyan, M. K. (2017). Defect detection and classification of electronic circuit boards using keypoint extraction and CNN features. In The Ninth International Conferences on Pervasive Patterns and Applications. Athens, Greece: laria.

Wang, L., Törngren, M., \& Onori, M. (2015). Current status and advancement of cyber-physical systems in manufacturing. Journal of Manufacturing Systems, 37(2), 517-527. http://dx.doi.org/10.1016/j.jmsy.2015.04.008.

Wang, T., Chen, Y., Qiao, M., \& Snoussi, H. (2018). Afast and robust convolutional neural network-based defect detection model in product quality control. International Journal of Advanced Manufacturing Technology, 94(9-12), 3465-3471. http://dx.doi. org/10.1007/s00170-017-0882-0.

Xu, L. D., Xu, E. L., \& Li, L. (2018). Industry 4.0: state of the art and future trends. International Journal of Production Research, 56(8), 2941-2962. http://dx.doi.org/10.1080/00207543.2018.1444806.

Xu, L., Lv, S., Deng, Y., \& Li, X. (2020). A weakly supervised surface defect detection based on convolutional neural network. IEEE Access: Practical Innovations, Open Solutions, 8, 42285-42296. http://dx.doi.org/10.1109/ACCESS.2020.2977821.

Yang, J., Li, S., Wang, Z., \& Yang, G. (2019). Real-time tiny part defect detection system in manufacturing using deep learning. lEEE Access: Practical lnnovations, Open Solutions, 7, 89278-89291. http://dx.doi.org/10.1109/ACCESS.2019.2925561.

Yang, S., Li, X., Jia, X., Wang, Y., Zhao, H., \& Lee, J. (2020). Deep learning-based intelligent defect detection of cutting wheels with industrial images in manufacturing. Procedia Manufacturing, 48, 902-907. http://dx.doi.org/10.1016/j.promfg.2020.05.128.

Yapi, D., Allili, M. S., \& Baaziz, N. (2017). Automatic fabric defect detection using learning-based local textural distributions in the contourlet domain. IEEE Transactions on Automation Science and Engineering, 15(3), 1017-1026.

$\mathrm{Yu}$, L., Wang, Z., \& Duan, Z. (2019). Detecting gear surface defects using background-weakening method and convolutional neural network. Journal of Sensors, 2019, 1-13. http://dx.doi.org/10.1155/2019/3140980.

Web of Science. (2021). Retrieved in 15 March 2021, from http://isiknowledge.com/.

Yuan, T., Bae, S. J., \& Park, J. 1. (2010). Bayesian spatial defect pattern recognition in semiconductor fabrication using support vector clustering. International Journal of Advanced Manufacturing Technology, 51(5-8), 671-683. http://dx.doi.org/10.1007/s00170010-2647-x.

Yuan, T., Kuo, W., \& Bae, S. J. (2011). Detection of spatial defect patterns generated in semiconductor fabrication processes. IEEE Transactions on Semiconductor Manufacturing, 24(3), 24. http://dx.doi.org/10.1109/TSM.2011.2154870.

Zhang, J., Lu, C., Wang, J., Wang, L., \& Yue, X. (2019). Concrete cracks detection based on FCN with Dilated convolution. Applied Sciences, 9(13), 2686. http://dx.doi.org/10.3390/app9132686.

Zhang, K., \& Shen, H. (2021). Solder joint defect detection in the connectors using improved faster RCNN algorithm. Applied Sciences, 11(2), 576. http://dx.doi.org/10.3390/app11020576.

Zhao, W., Huang, H., Li, D., Chen, F., \& Cheng, W. (2020a). Pointer defect detection based on transfer learning and improved cascadeRCNN. Sensors, 20(17), 4939. http://dx.doi.org/10.3390/s20174939. PMid:32882801.

Zhao, S., Yin, L., Zhang, J., Wang, J., \& Zhong, R. (2020b). Real-time fabric defect detection based onmulti-scale convolutional neural network. IET Collaborative Intelligent Manufacturing, 2(4), 189-196. http://dx.doi.org/10.1049/iet-cim.2020.0062.

Zhao, W., Chen, F., Huang, H., Li, D., \& Cheng, W. (2021). A new steel defect detection algorithm based on deep learning. Computational Intelligence and Neuroscience, 2021, 5592878. http://dx.doi.org/10.1155/2021/5592878. PMid:33824656.

Zhu, Y., Yang, R., He, Y., Ma, J., Guo, H., Yang, Y., \& Zhang, L. (2021). A lightweight multiscale attention semantic segmentation algorithm for detecting laser welding defects on safety vent of power battery. IEEE Access : Practical Innovations, Open Solutions, 9, 39245-39254. http://dx.doi.org/10.1109/ACCESS.2021.3064180. 